\title{
Geschlechtsunterschiede bei psychologischen, ernährungs- und sportwissenschaftlichen Einflussfaktoren auf Adipositas/Übergewicht bei Kindern und Jugendlichen in Österreich
}

\author{
Elisabeth Ardelt-Gattinger · Susanne Ring-Dimitriou • Johannes Hofmann · Katharina Paulmichl · \\ Fanni Zsoldos · Daniel Weghuber
}

Eingegangen: 9. Oktober 2015 / Angenommen: 23. Dezember 2015 / Online publiziert: 5. Februar 2016

(C) Die Autor(en) 2016. Dieser Artikel ist auf Springerlink.com mit Open Access verfügbar

Zusammenfassung Das Ess- und Bewegungsverhalten unterliegt der Steuerungsfunktion durch bestimmte kognitive Muster. Es werden im Folgenden, zur Darstellung von eventuell durchgehenden Geschlechtertrends, die bei 4400 Kindern/Jugendlichen und 6600 Erwachsenen mit dem Adipositas Diagnostik- und Evalutionssystem (AD-EVA) gemessenen Unterschiede in der Teilgruppe der Adipösen dargestellt.

Höhere Werte ungünstiger psychischer Steuerungsmechanismen des Ess- und Bewegungsverhaltens adipöser Mädchen liegen vor allem im Bereich der vorklinischen Essstörungen. Diese Ergebnisse sind für Prävention und Gesundheitsförderung relevant.

Interessant sind auch die nicht-signifikanten Unterschiede im Bereich der Einstellung zu Bewegung wie der Bewegungsmotivation. Es würde bedeuten, dass man nicht dem Vorurteil aufsitzen darf, dass das männliche Geschlecht ohnehin mehr Freude an der Bewegung habe.

Priv.-Doz. Dr. D. Weghuber $(\bowtie) \cdot$ J. Hofmann · K. Paulmichl · F. Zsoldos

Universitätsklinik für Kinder- und Jugendheilkunde,

Paracelsus Medizinische Privatuniversität,

Müllner Hauptstraße 48,

5020 Salzburg, Österreich

E-Mail: d.weghuber@salk.at

E. Ardelt-Gattinger $\cdot$ J. Hofmann

Fachbereich Psychologie, Universität Salzburg,

Salzburg, Österreich

J. Hofmann · E. Ardelt-Gattinger · Priv.-Doz. Dr. D. Weghuber Obesity Academy Austria,

Salzburg, Österreich

S. Ring-Dimitriou

Interfakultärer Fachbereich für Sport- und

Bewegungswissenschaften, Universität Salzburg,

Salzburg, Österreich
Die männliche Vorliebe für „Snacks“ und „Deftiges“ konnte in der für Österreich repräsentativen Normstichprobe des Fragebogensystems AD-EVA repliziert werden, spiegelt sich aber nicht in der Teilgruppe der adipösen Mädchen und Knaben. Man kann davon ausgehen, dass sich 8- bis 18-jährige Adipöse beiderlei Geschlechts ungünstig ernähren.

Schlüsselwörter Geschlechtsunterschiede · Adipositas · Einflussfaktoren • Ernährungsverhalten • Sportmotivation $\cdot$ Kinder $\cdot$ Erwachsene

Gender differences of psychological, nutritional, and physical fitness variables influencing obesity/overweight in Austrian children and adolescents

Summary Eating behavior and physical activity behavior are under the control of certain cognitive patterns. 6600 adults and 4400 children/adolescents (8-18 years) were tested with the Obesity Diagnostics and Evaluation System (AD-EVA). Potentially significant gender differences will be detailed for the entire juvenile cohort, the subgroup of obese children/adolescents as compared to the adult cohort in this article.

Among all the subscales tested, obese girls primarily showed significantly higher values of (preclinical) eating disorders than boys. These data are relevant for both prevention and health promotion.

No significant differences were found in regard to sports motivation. This warrants facilitation of physical activity for both genders. Further, a male predilection for "Snacks" and "High-fat food" that could be found in the total representative study group, could not be verified in the subgroup of obese girls and boys, thus suggesting a similarily unhealthy eating behavior in both genders of juvenility. 
Keywords Gender differences · Obesity · Impact factors . Eating behavior - Sports motivation - Children · Adults

\section{Einleitung}

Adipositas wird nicht als psychische Störung, sondern eindeutig als chronische Krankheit bezeichnet, die allerdings primär durch ein bestimmtes Ess- und Bewegungsverhalten beeinflusst wird. Dieses wiederum unterliegt wie jedes andere Verhalten der Steuerungsfunktion durch bestimmte kognitive Muster. Zudem kann es durch eine massiv stigmatisierende Umwelt und durch komplexe Rückkoppelungen zu Komorbiditäten kommen [1].

Literatur über Geschlechtsunterschiede liegt nur für wenige der genannten Verhaltensweisen bzw. deren kognitiven Steuerungsvariablen vor: Auf der Basis des bereits 1985 von Stunkard \& Messick [2] entwickelten und von Pudel \& Westenhöfer [3] für die deutsche Sprache adaptierten Fragebogens zum Essverhalten (FEV), in einer Revision in Fragebogen zum pathogenen Essverhalten umbenannt (FEV-path) [4], berichten die letztgenannten Autoren über höhere Kognitive Kontrolle des Essverhaltens bei Frauen, häufig auch als „restraint eating “ bezeichnet [3]. Dies stimmt mit der höheren Prävalenz sowohl für vorklinische Essstörungen im Sinne der ,Überbesorgtheit um Figur und Äußeres‘ wie für das Vollbild von Essstörungen, Bulimie, bei Frauen überein [5]. Männer werden in bezug auf unkontrollierte Essanfällen, die sog. Binge Eating Disorder, allerdings als gefährdeter eingestuft [6]. Bezüglich der salutogenen Faktoren wie „Empfehlungen für gesundes Leben umsetzen“, „Genuss am Essen“ oder "grundsätzliche positive Einstellung zu Bewegung im Sport" liegen speziell für adipöse Menschen noch keine Daten vor.

Der Suchtaspekt übermäßigen Essens ist durch neurokognitive Forschungen und Fragebogenuntersuchungen unumstritten, Unterschiede bestehen aber in der Auffassung, ob es sich um Sucht nach bestimmten Nahrungsbestandteilen oder -mitteln (carb-craving, Schokolade-Abhängigkeit etc.) handelt [7, 8]. Da es keine eindeutige Datenlage gibt, manches auch mit Präferenzen für bestimmte Nahrungsmittel interagiert, wird für die vorliegende Studie von einem allgemeinen ,Craving nach und Abhängigkeit von übermäßigem Essen' ausgegangen [9].

Vermutete Geschlechtsunterschiede im Bereich der Bewegungsmotivation gehen in die Richtung, dass die „extrinsische“ bei Frauen höher vermutet wird [10], während die „intrinsische Sportmotivation “ beim männlichen Geschlecht stärker sein sollte [11], was auch eine „positive Einstellung zu Bewegung im Sport“ nahe legt.

Die schlechte Lebensqualität Adipöser aller Altersklassen ist bekannt [12]. In einer Auswertung des Kinder- und Jugendgesundheitssurveys, zu der auch der KINDL-Fragebogen zur Lebensqualität gehörte, berichten Kurt \& Ellert [13], dass adipöse Kinder/Jugendliche in Bezug auf die Subskalen „familiäre Lebens- qualität“ und "psychisches Wohlbefinden" keine geschlechtsspezifischen Unterschiede aufweisen. Wohl aber sei das „körperliche Wohlbefinden“ und der „Selbstwert" von Mädchen stärker beeinträchtigt als jenes/r von Jungen. Letztere geben aber eine schlechtere Lebensqualität im Bereich „Freunde“ an. Es wird aber von Kurth und Ellert [13] darauf hingewiesen, dass die Wahrnehmung des eigenen Körperumfangs als mehr oder weniger dick einen starken Einfluss auf die Lebensqualität hat.

In der für Gesundheit und Übergewicht günstigen Vorliebe für "gesunde“ Ernährung unterscheiden sich weder Geschlechter noch Gewichtsklassen nach der bisherigen Literatur im Kindesalter nicht $[14,15]$. Übereinstimmend wird aber berichtet, dass erwachsene Frauen "gesündere" Ernährung und weniger deftig-fette Nahrung bevorzugen als Männer [5].

\section{Ziele}

Ziel der vorliegenden Studie ist eine zwischen Normalgewicht und Adipositas differenzierende Darstellung von Geschlechtsunterschieden bei den in Abhängigkeit vom Gewicht variierenden Steuerungsvariablen des Essund Bewegungsverhaltens von Kindern/Jugendlichen zu erheben.

\section{Methoden}

Die Testung erfolgte mit einem eigens für Diagnostik und Evaluation von Adipositas und anderen Lebensstilerkrankungen konstruierten Fragebogensystem. Dieses wurde an einer für Österreich repräsentativen Gesamtstichprobe von 6600 Erwachsenen und 4400 Kindern/Jugendlichen zwischen 8 und 18 entwickelt, teststatistisch auf Testgenauigkeit (Cronbach Alfa) und Test-Retestkonstanz geprüft und validiert [4]. Es misst ,pathogenes' Essverhalten (FEV_path) und es wurde - im Sinne salutogener Steuerung des Ess- und Bewegungsverhaltens - auch ein diesbezüglicher Fragebogen (FEVsalut) mit den Subskalen „Empfehlungen umsetzen“ und „Grundeinstellung gegenüber Bewegung und Sport" hinzugefügt. Dem Suchtaspekt übermäßigen Essens wurde durch einen Fragebogen (FUN), der das eingangs zitierte ,Craving nach und Abhängigkeit von übermäßigem Essen' misst, Rechnung getragen. Bewegungsmotivation (FBM) wurde sowohl intrinsisch (Bewegung aus Spaß und Freude) wie extrinsisch (Bewegung um schön und schlank zu werden) erhoben. Anstelle der meist verwendeten Häufigkeitsangaben der eingenommen Nahrungsmittel wurde ein Konstrukt operationalisiert, das bessere Voraussagen für stabile Voraussagen der Änderung der Essgewohnheiten zulässt, nämlich eine Ernährungspräferenzenliste mit den Subskalen „Gesundes“, „Deftiges“ und „Snacks“.

Da Essstörungen auch in der Jugend zu den häufigen Komorbiditäten von Adipositas zählen [16], wurden diese in ihren klinischen Vollbildern Bulimie und Binge Eating 
Disorder erfasst und durch einen Fragebogen zu vorklinischen Essstörungen, der sog. „Preoccupation with weight and shape" und der vorklinischen Bulimie [4]. Vorklinische Bulimie bedeutet, dass das Erbrechen nach kleinen Essenmengen erfolgt, bei denen das Gefühl vorherrscht, mehr gegessen zu haben, als für die Wunschfigur ,erlaubt' gewesen wäre, also ein ,ab und an Erbechen' auch nach Einnahme subjektiv als zu groß empfundener Nahrungsmengen. Schließlich wurde die Lebensqualität der Kinder und Jugendlichen durch den sechs verschiedene Lebensbereiche erfassenden KINDL [12] gemessen.

\section{Stichprobe}

Die Auswahl der Versuchspersonen ist mit der Stichprobe der Testentwicklung und Eichung der Fragebogenbatterie AD-EVA an 6600 für Österreich repräsentativen Kindern und Jugendlichen zwischen 8 und 18 Jahren ident [4], wobei nicht immer alle 8 Fragebögen bzw. deren 13 Subskalen von allen ausgefüllt wurden, da meist in Schulen mit beschränktem Zeitbudget getestet wurde. Da bei dieser Untersuchung eine Perzentilgruppe der „morbid Untergewichtigen“ (unterste 0,5\%) interpoliert wurde, sind die Adipositasgruppen das 8. und 9. Perzentil (oberste 2,5 und 0,5\%).

\section{Ergebnisse}

Die Suchtwerte übermäßigen Essens unterscheiden die Geschlechter weder in der Gesamt- noch in der Subgruppe der Adipösen (vgl. Tab. 1 und 2).
Mädchen zeigen über alle Gewichtsgruppen der 8bis 18-Jährigen hinweg sehr signifikant höhere Werte des Pathogenen Essverhaltens „Ess-Kontrolle“ (Restraint Eating) und des sog. „Emotionsessens“, diese Ergebnisse erreichen auch in der Teilstichprobe adipöser Kinder/ Jugendlicher noch Signifikanz. Gar kein Unterschied fand sich für die „Disinhibition“, die „Verführbarkeit durch Geruch und Anblick von Speisen“ (vgl. Tab. 1 und 2).

Auf dem „Fragebogen zum salutogenen Ess- und Bewegungsverhalten" sind die Unterschiede zwischen Mädchen und Jungen aller Gewichtsklassen nur in „Empfehlungen umsetzen“ signifikant, aber auch hier mit niedriger Effektstärke. Für die Subgruppe der adipösen Kinder/Jugendlichen sind keine Geschlechterdifferenzen mehr nachweisbar. Die zweite Subskala, die "grundsätzliche Einstellung zu Sport/Bewegung" misst, weist in keiner der Teilstichproben Unterschiede auf (vgl. Tab. 1 und 2).

Die durch die Subskalen „Spaß und Befriedigung“ (intrinsische Bewegungsmotivation) und „Erfolg durch Leistung und Ästhetik“ (extrinsische Bewegungsmotivation) des AD-EVA überprüften Unterschiede waren weder bei der Gesamtheit aller Kinder/Jugendlichen Gewichtsgruppen noch bei der Teilgruppe der Adipösen signifikant (vgl. Tab. 1 und 2).

Bezüglich der Präferenzen für „Deftiges“ und „Snacks“ liegen Jungen aller Gewichtsklassen signifikant und substanziell höher als Mädchen. Dieser Geschlechterunterschied verschwindet aber bei der Gruppe Adipöser (vgl. Tab. 1 und 2). In derVorliebe für „gesunde“ Ernährung unterscheiden sich die Geschlechter unabhängig vom Gewicht nicht (vgl. Tab. 1 und 2).

Die Lebensqualität weist keinen Gendereffekt auf.

Tab. 1 Geschlechtsunterschiede den kognitiven Steuerungsvariablen des Ess- und Bewegungsverhaltens, der Lebensqualität und der Essstörungen in der AD-EVA Normstichprobe und bei Adipösen und Morbid Adipösen

\begin{tabular}{|c|c|c|c|c|c|c|c|c|c|}
\hline \multirow[b]{2}{*}{ Fragebogen } & \multirow[b]{2}{*}{ Subskalen } & \multicolumn{4}{|c|}{ Normstichprobe Perzentile 1-9 } & \multicolumn{4}{|c|}{ Perzentile 8\&9 Adipositas } \\
\hline & & $\mathrm{F}$ & $p$ & $\eta 2$ & $\mathrm{df} \mathrm{m} / \mathrm{w}$ & $\mathrm{F}$ & $p$ & $\eta 2$ & $\mathrm{df} \mathrm{m} / \mathrm{w}$ \\
\hline FUN & Craving und Abhängigkeit & & n.s. & & & & n.s. & & \\
\hline \multirow[t]{3}{*}{ FEV-Path } & Kogn. Kontrolle & 31,85 & $<0,01$ & 0,02 & $999 / 874$ & 4,07 & $<0,05$ & 0,003 & $60 / 70$ \\
\hline & Disinhibition & & n.s. & & & & n.s. & & \\
\hline & Emotionsessen & 17,66 & $<0,01$ & 0,009 & $989 / 870$ & 3,64 & $<0,05$ & 0,03 & $59 / 74$ \\
\hline \multirow[t]{2}{*}{ FEV-Salut } & Empfehlungen umsetzen & 26,96 & $<0,01$ & 0,03 & $747 / 812$ & & n.s. & & \\
\hline & Pos. Einstellung zu Bewegung & & n.s. & & & & n.s. & & \\
\hline FBM & Bewegmotivation & & n.s. & & & & n.s. & & \\
\hline \multirow[t]{3}{*}{ EPL } & Snacks süß und fett & 31,27 & $<0,01$ & 0,07 & $774 / 909$ & & n.s. & & \\
\hline & Gesundes & & n.s. & & & & n.s. & & \\
\hline & Fettes, Deftiges & 40,42 & $<0,01$ & 0,07 & $772 / 899$ & & n.s. & & \\
\hline LQS & Lebensqualität & & n.s. & & & & n.s. & & \\
\hline FVE & Präklin. Essstörung & 77,97 & $<0,01$ & 0,04 & $827 / 929$ & 7,33 & $<0,01$ & 0,06 & $109 / 163$ \\
\hline \multirow[t]{2}{*}{ FBEB } & Bulimie & 10,11 & $<0,01$ & 0,008 & $560 / 668$ & 2,89 & $<0,01$ & 0,02 & $98 / 113$ \\
\hline & BED & 18,77 & $<0,01$ & 0,02 & $561 / 666$ & 3,19 & $<0,01$ & 0,04 & $89 / 114$ \\
\hline \multicolumn{10}{|c|}{$\begin{array}{l}\text { Fragebogen (FB) zum Umgang mit Nahrungsmitteln (Suchtfragebogen), FEV_path FB zum pathogenen Essverhalten, FVE_salut FB zum salutogenen Essver- } \\
\text { halten, FBM FB zur Bewegungsmotivation, EPL Ernährungspräferenzenliste LQS Lebensqualitäts-Skala, FVE Fragebogen zu vorklinischen Essstörungen, } F B E B \\
\text { Fragebogen zu Big Eating, Bulimie und Binge Eating Disorder; } F=\text { Test Statistic: Univariate ANOVAs. Fett gedruckt = signifikante Unterschiede zwischen den } \\
\text { Geschlechtern }\end{array}$} \\
\hline
\end{tabular}


Tab. 2 Deskriptive Werte der Geschlechtsunterschiede in den kognitiven Steuerungsvariablen des Ess- und Bewegungsverhaltens, der Lebensqualität und der Essstörungen in der AD-EVA Normstichprobe und bei Adipösen und Morbid Adipösen

\begin{tabular}{|c|c|c|c|c|c|c|c|c|c|}
\hline \multirow[b]{3}{*}{ Fragebögen } & \multirow[b]{3}{*}{ Subskalen } & \multicolumn{4}{|c|}{ Normstichprobe Perzentile 1-9 } & \multicolumn{4}{|c|}{ Perzentile 8\&9 Adipositas } \\
\hline & & \multicolumn{2}{|l|}{ männl } & \multicolumn{2}{|l|}{ weibl } & \multicolumn{2}{|l|}{ männl } & \multicolumn{2}{|l|}{ weibl } \\
\hline & & M & SD & $M$ & SD & M & SD & M & SD \\
\hline FUN & Craving und Abhängigkeit & & & & & & & & \\
\hline \multirow[t]{3}{*}{ FEV-path } & Kontrolle & 24,01 & 10,56 & 27,15 & 11,29 & 31,52 & 10,56 & 35,02 & 9,97 \\
\hline & Disinhibition & & & & & & & & \\
\hline & Emotionsessen & 12,08 & 5,00 & 13,94 & 5,67 & 14,51 & 6,23 & 18,77 & 7,20 \\
\hline \multirow[t]{2}{*}{ FEV-salut } & Empfehlungen umsetzen & 27,61 & 6,58 & 28,91 & 5,47 & & & & \\
\hline & Einstellung zu Bewegung & & & & & & & & \\
\hline FBM & Bewegungsmotivation & & & & & & & & \\
\hline \multirow[t]{3}{*}{ EPL } & Snacks fett, süß & 89,50 & 20,10 & 80,51 & 19,00 & & & & \\
\hline & Gesundes & & & & & & & & \\
\hline & Fettes, Deftiges & 26,06 & 6,24 & 23,01 & 6,14 & & & & \\
\hline SLQ & Lebensqualität & & & & & & & & \\
\hline FVE & Vorklinische Essstörung & 17,03 & 6,56 & 20,02 & 7,35 & 21,87 & 5,07 & 25,32 & 5,81 \\
\hline \multirow[t]{3}{*}{ FBEB } & Bulimie & 6,71 & 11,35 & 8,71 & 13,56 & 10,67 & 13,57 & 14,31 & 16,13 \\
\hline & Binge Eating & 3,40 & 5,67 & 5,01 & 7,05 & 8,60 & 8,29 & 10,28 & 8,41 \\
\hline & BIG Eating & & & & & & & & \\
\hline
\end{tabular}

In den vorklinischen Essstörungen, die für Kinder/ Jugendliche sowohl das „preoccupied with weight and shape" und das zusätzliche Item „vorklinische Bulimie“ oder „bulimisches Probierverhalten“ enthalten, ebenso wie bei den Vollbildern der Bulimie und Binge Eating Disorder, liegen Mädchen sehr signifikant höher. Dieser Unterschied ist auch bei den adipösen Kindern/Jugendlichen sehr signifikant und substantiell.

\section{Interpretation}

Das von vielen Adipösen geschilderte „Craving nach und Abhängigkeit von übermäßigem Essen " und Publikationen, die Vergleiche mit anderen Abhängigkeiten (Sport, Alkohol, Nikotin, Drogen) nachweisen konnten [17, 18], unterstützten den Ansatz „übermäßiges Essen“ als Sucht bedingt zu betrachten. Neuere Literatur [7, 19] attestiert übermäßigem Essen, wie es bei Adipösen auftritt, bereits einhellig Suchtcharakter. Diese "Sucht“- werte unterscheiden weder die Gesamtgruppe der 8- bis 18-Jährigen noch die adipösen Mädchen und Jungen voneinander. Bei ersteren hat diese ,Sucht' wenig Relevanz, bei den Adipösen erscheint es plausibel, dass dieses primär auch hormonelle Geschehen [17] in bezug auf Geschlecht keine Unterschiede aufweist.

Der Trend zu erhöhtem „restraint eating“ und höherem ,Emotionsessen“ der Mädchen und den ebenfalls höheren Werten für Essstörungen entspricht der einschlägigen Literatur, die es in Zusammenhang mit dem - gefährlichen - dünnen weiblichen Schönheitsideal setzt [6, 20-22]. Auch auf dem im Gegensatz zum pathogenen Zugang der oben dargestellten Konstrukte entwickelten „Fragebogen zum salutogenen Ess- und
Bewegungsverhalten " zeigen Mädchen in höherem Ausmaß, dass sie die für gesundes Leben und schlanke Figur in allen Medien gegebenen „Empfehlungen“ besser „umsetzen“ können [5].

Die Subskala des FEV-salut „grundsätzliche Einstellung zu Sport/Bewegung “ waren weder bei der Gesamtheit aller Kinder/Jugendlichen Gewichtsgruppen noch bei der Teilgruppe der Adipösen signifikant. Das geht konform mit den ebenfalls gleichen Ergebnissen zur Bewegungsmotivation „Spaß und Befriedigung“ (intrinsisch) und „Erfolg durch Leistung und Ästhetik“ (extrinsisch). Es spricht gegen die Meinung, der einschlägigen Genderforschung und -Praxis, dass Jungen motivierter zu Bewegung und Sport seien [23]. Die Tatsache, dass Jungen aller Perzentilgruppen signifikant und substanziell höher bei „Deftiges“ und "Snacks“ liegen und dieser Geschlechterunterschied nur bei der Gruppe Adipöser verschwindet, spricht sogar für eine verstärkte Gesundheitsförderung bei Jungen.

Die Vorliebe für "gesunde" Ernährung unterscheidet die Geschlechter konform mit der Literatur $[14,15]$ im Kindesalter nicht und verweist auf die - auch Stigmatisierungen der ,Dicken' vorbeugende - Sinnhaftigkeit der gemeinsamen salutogene Prävention ungünstigen Essverhaltens.

Der Gesamtwert der mit dem KINDL gemessenen Lebensqualität in der Subgruppe Adipöser Kinder/ Jugendlicher unterscheidet die Geschlechter in keiner der untersuchten Gruppen, was in Bezug auf „psychisches Wohlbefinden“ der in einer größeren Stichprobe von Ravens-Sieberer et al. [12] erzielten Werte entspricht. Für die Subgruppe der Adipösen stimmen die Ergebnisse mit der Darstellung von Kurth und Ellert [13] überein, dass die Wahrnehmung des eigenen Körperumfangs als mehr 
oder weniger dick einen hauptsächlichen Einfluss auf die Lebensqualität hat.

Bezüglich der häufigsten psychischen Komorbidität der Adipositas, den vorklinischen und klinischen Essstörungen, geht man davon aus, dass sich das weibliche Geschlecht, insbesondere auch adipöse Frauen und Mädchen, mehr Sorgen um Figur und Äußeres macht $[1,4]$. Auch im Gesamten der Kinder-/JugendlichenStichprobe liegt der genannte Geschlechterunterschied für diese Skala vor. Mädchen liegen signifikant und substantiell höher in Bulimie und Binge Eating Disorder. Er ist bei den adipösen Kindern/Jugendlichen ebenfalls sehr signifikant und erreicht die höchste Effektstärke der berechneten Unterschiede.

In der Literatur wird einheitlich von einem höheren Essstörungsrisiko für Frauen gesprochen, das auch im DSM IV dokumentiert ist [20]. Dies gilt sowohl für Bulimie wie für Binge Eating Disorder [6]. Daten über Geschlechterdifferenzen weisen allerdings einen zeitlichen Wandel auf. So wird die Prävalenz für Bulimie für das weibliche Geschlecht [21, 22] als höher angegeben, viele ältere Fragebögen enthalten aber etwa ,exzessiven Sport' nicht als bulimische Kompensationsmöglichkeit, welche nach einer Untersuchung von Lewinsohn et al. [16] bei Männern wesentlich öfter angewandt wird, Männer zudem wesentlich seltener Therapien aufsuchen, was etwa bei Befragungen von TherapeutInnen zu Fehleinschätzungen führen kann. Declan, Grilo, \& Masheb [24] berichten in ihrer Studie von einem höheren Auftreten von BED bei Männern. Sie verweisen auch auf die Tatsache, dass es auch innerhalb einer bestimmten Essstörung relevante Geschlechterunterschiede gibt, wie etwa bezüglich der Gründe für Schamgefühle über ,Fressanfälle‘ oder des vermehrten Drogenkonsums in der Anamnese von Männern mit Binge Eating Disorder.

In einer Studie von Vincent \& McCabe [25] wurden für 11- bis 17-jährige Mädchen und Jungen keine signifikanten Unterschiede in Bezug auf BED und Bulimie gefunden, wohl aber bewirkten häufige Diskussionen über Gewichtsabnahme mit Vätern ein höheres Essstörungsrisiko bei Mädchen, jene mit Müttern ein höheres der Jungen, ein Gendereffekt, der bislang wenig untersucht wurde und wenig Berücksichtigung fand.

Zusammenfassend lässt sich sagen, dass Unterschiede, d. h. höhere Werte ungünstiger psychischer Steuerungsmechanismen des Ess- und Bewegungsverhaltens adipöser Mädchen, vor allem im Bereich der Essstörungen liegen. Diese Ergebnisse sind für Prävention und Gesundheitsförderung relevant. Sie unterstützen die Kritik an dem auf der Makroebene etablierten dünnen weiblichen Schönheitsideal. Ähnliche Werte in den Vollbildern der Essstörungen und faires Gendern sollte auch die Forcierung der ,Waschbrett'-Männlichkeit, ja allgemein ungesunder „Konstruktion von Geschlechtlichkeit“ [26] hinterfragen. Auf alle Fälle sind Forderungen nach frühen und gemeinsamen Interventionen gegen Essstörungen und Adipositas [1] zu unterstützen.

Interessant sind freilich auch die nicht signifikanten Unterschiede im Bereich der Einstellung zu Bewegung wie der Bewegungsmotivation. Es würde bedeuten, dass die Förderung von adipösen Jungen und Mädchen für beide Geschlechter gleich wichtig ist und man nicht dem Vorurteil aufsitzen darf, dass das männliche Geschlecht ohnehin mehr Freude an der Bewegung habe [23].

Die männliche Vorliebe für „Snacks“ und „Deftiges“, konnte in der für Österreich repräsentativen Normstichprobe des Fragebogensystems AD-EVA repliziert werden [4] und spiegelt sich auch in der Teilgruppe der adipösen Mädchen und Knaben. Man kann davon ausgehen, dass sich 8- bis 18-jährige Adipöse beiderlei Geschlechts ungünstig ernähren, d. h. das Bild der in vielen bildhaften Darstellungen verankerten ,weiblichen' Kühlschränke mit viel Obst, Gemüse und wenig Fettem und Deftigem [27] auf adipöse Kinder und Jugendliche nicht zutrifft.

\section{Einhaltung ethischer Richtlinien}

\section{Interessenkonflikt und Ethik}

Elisabeth Ardelt-Gattinger, Susanne Ring-Dimitriou, Johannes Hofmann, Katharina Paulmichl, Fanni Zsoldos und Daniel Weghuber geben an, dass kein Interessenkonflikt besteht. Eine schriftliche Zustimmung der Patienten, bei Minderjährigen auch eines Elternteiles, wurde gemäß den Richtlinien der Deklaration von Helsinki von 1975 ebenso wie ein positives Votum der lokalen Ethikkommission (Salzburg) eingeholt.

\section{Open Access}

Dieser Artikel unterliegt den Bedingungen der Creative Commons Attribution License. Dadurch sind die Nutzung, Verteilung und Reproduktion erlaubt, sofern der/die Originalautor/en und die Quelle angegeben sind.

\section{Literatur}

1. Ardelt-Gattinger E, Ring-Dimitriou S, Weghuber D, Herausgeber. Der gesunde Adipöse. Das Kontinuum zwischen gesunder und kranker Adipositas. Bern: Huber; 2015.

2. Stunkard AJ, Messick S. The three-factor eating questionnaire to measure dietary restraint, disinhibition and hunger. J Psychosom Res. 1985;29:71-83.

3. Pudel V, Westenhöfer J. Fragebogen zum Essverhalten. Göttingen: Hogrefe; 1989.

4. Ardelt-Gattinger E, Meindl M, Herausgeber. Interdisziplinäres Testsystem zur Diagnostik und Evaluation bei Adipositas und anderen durch Ess- und Bewegungsverhalten beeinflussbaren Krankheiten (AD-EVA). Bern: Huber; 2010.

5. Huon G, Lim J. The emergence of dieting among female adolescents: age, body mass index, and seasonal effects. Int J Eat Disord. 2000;28(2):221-5.

6. de Zwaan M, Friederich HC. Binge Eating Störung. Ther Umschau. 2006;63(8):529-33.

7. Wang G, Volkow N, Thanos P, et al. Similarity between obesity and drug addiction as assessed by neuro functional imaging. J Addict Dis. 2004;23(3):39-53.

8. Weiner R. Gendermedizinische Differenzen beeinflussen OP Erfolg. Adipositas Spektrum. 2013;9(1):4. 
9. Ardelt-Gattinger E, Lechner H. Dependency of smokers, alcoholics and obese patients. Poster auf der Tagung „Obesity“. Int J Obes. 2000.

10. Pratscher HSportverhalten in Österreich.JErnährungsmed. 2000;2(5):18-23.

11. Biddle S, Fox K. Motivation for physical activity and weight management. Int J Obes. 1998;22(2):39-47.

12. Ravens-Sieberer U. Verfahren zur Erfassung der gesundheitsbezogenen Lebensqualität bei Kindern und Jugendlichen. Bundesgesundheitsblatt - Gesundheitsforschung - Gesundheitsschutz. 2000;43:198-209.

13. Kurth BM, Eller U. Gefühltes oder tatsächliches Übergewicht: Worunter leiden Jugendliche mehr? Ergebnisse des Kinder- und Jugendgesundheitssurveys KiGGS. Dtsch Arztebl. 2008;105(23):406-12.

14. Koletzko B, Dokoupil K, Reitmayr S, et al. Dietary fat intakes in infants and primary school children in Germany. Am J Clin Nutr. 2002;72:1392S-8S.

15. Maffeis C, Schutz Y, Chini L, et al. Effects of dinner composition on postprandial macronutrient oxidation on pre pubertal girls. Obes Res. 2004;12(7):1128-35.

16. Lewinsohn PM, Seeley JR, Moerk KC, et al. Gender differences in eating disorder symptoms in young adults. Int J Eat Disord. 2002;32(4):426-40.

17. Ardelt-Gattinger E, Meindl $M$, Mangge $H$. Beeinflusst bariatrische Chirurgie Sucht und Essstörungen? Chirurg. 2012;83:561-7.

18. Ardelt-Gattinger E, Lengenfelder P, Lechner H. Evaluation interdisziplinär vernetzter Adipositas-Therapie unter Berücksichtigung der Suchtkomponenten. VPP. 2003;35:735-68.
19. Davis C, Curtis C, Levitan RD, et al. Evidence that ,food addiction' is a valid phenotype of obesity. Appetite. 2011;57:711-7.

20. Ihle W, Laucht M, Schmidt MH, Esser G. Geschlechtsunterschiede in der Entwicklung psychischer Störungen. Gehirn und Geschlecht. New York: Springer; 2007.

21. de Zwaan M, Schüssler P. Diagnostik und Therapie der Anorexia und Bulimia nervosa. J Ernährungsmed. 2000;1:16-9.

22. Kinzl F. Essstörungen bei Männern und Frauen: Eine Repräsentativerhebung. ZErnährungswiss. 1998;37:336-42.

23. Ring-Dimitriou S, Ardelt-Gattinger E, Gattinger E. FBM Fragebogen zur Bewegungsmotivation. In: Ardelt-Gattinger E, Meindl M, Herausgeber. AD-EVA. Interdisziplinäres Testsystem zur Adipositas-Diagnostik und Evaluation (ADEVA). Bern: Huber; 2010.

24. Declan TB, Grilo C, Masheb M. Gender differences in patients with binge eating disorder. Int $\mathrm{J}$ Eat Disord. 2001;31(1):63-70.

25. Vincent MA, McCabe MP. Gender differences among adolescents in family, and peer influences on body dissatisfaction, weight loss, and binge eating behaviors. J Youth Adolesc. 2000;29(2):205-21.

26. Kolip P. Geschlecht und Gesundheit im Jugendalter. Die Konstruktion von Geschlechtlichkeit über somatische Kulturen. Opladen: Leske + Budrich; 1997.

27. Wardle J, Haase AM, Steptoe A, et al. Gender differences in food choice: the contribution of health beliefs and dieting. Ann Behav Med. 2004;27(2):107-16. 